\title{
How Powerful is Demography? The Serendipity Theorem Revisited*
}

\author{
David de la Croix ${ }^{\dagger} \quad$ Pierre Pestieau $^{\ddagger} \quad$ Grégory Ponthière ${ }^{\S}$
}

October 2010

\begin{abstract}
Introduced by Samuelson (1975), the Serendipity Theorem states that the competitive economy will converge towards the optimum steady-state provided the optimum fertility rate is imposed. This paper aims at exploring whether the Serendipity Theorem still holds in an economy with risky lifetime. We show that, under general conditions, including a perfect annuity market with actuarially fair return, imposing the optimum fertility rate and the optimum survival rate leads the competitive economy to the optimum steady-state. That Extended Serendipity Theorem is also shown to hold in economies where old adults work some fraction of the old-age, whatever the retirement age is fixed or chosen by the agents.
\end{abstract}

Keywords: Serendipity Theorem, fertility, mortality, overlapping generations, retirement.

JEL codes: E13, E21, I18, J10.

\footnotetext{
${ }^{*}$ The authors are grateful to Raouf Boucekkine, Hyppolite d'Albis, Julio Davila, Wolfgang Kuhle, Bertrand Wigniolle and two anonymous referees for their helpful comments on this paper.

${ }^{\dagger}$ IRES, CORE, UCLouvain, Belgium. E-mail: david.delacroix@uclouvain.be. The first author acknowledges the financial support of the Belgian French speaking community (Grant ARC "Sustainability") and the Belgian Federal Government (Grant PAI P6/07 Glonomics).

${ }^{\ddagger}$ University of Liege, CORE, Paris School of Economics and CEPR. E-mail: p.pestieau@ulg.ac.be.

${ }^{\S}$ Ecole Normale Supérieure, Paris School of Economics, France. E-mail: gregory.ponthiere@ens.fr.
} 


\section{Introduction}

Despite significant differences across countries, it is unquestionable that most economies have, during the last decades, converged from a demographic equilibrium characterized by a (relatively) high fertility and a (relatively) low longevity to another equilibrium, with a lower fertility and a higher longevity. Much attention has been paid, within economics, to that evolution. In particular, growth theory has, in the recent years, regarded the demographic equilibrium as an output to be explained, and built up several models aimed at explaining the demographic transition. ${ }^{1}$

However, there is also another way to look at the demography of an economy. Actually, one may treat demographic variables as inputs, whose levels can affect economic outcomes significantly. Indeed, given that fertility and mortality are major determinants of the functioning of an economy (e.g. savings, labour supply, etc.), one may think about their capacity to allow an economy to reach more or less high standards of living. More precisely, demographic variables can be regarded as powerful instruments to be influenced.

Such an alternative view leads to the following problem. Suppose that demographic variables could be controlled perfectly by a government. ${ }^{2}$ Then, two questions arise. First, to which values should those demographic variables be fixed? Second, if those demographic variables can be fixed optimally, to what extent would these allow a decentralized economy to reach the social optimum? In other words, would a perfect control of demographic variables lead a market economy to the optimum?

The second question was first asked and answered by Samuelson (1975), who highlighted, by means of his Serendipity Theorem, the power of demography. Actually, the Serendipity Theorem states that, if there exists a unique stable steady-state equilibrium in a Diamondtype overlapping generations (OLG) economy, a competitive economy will converge towards the most golden rule steady-state provided the optimum fertility rate is imposed. ${ }^{3}$ That theoretical result reveals the capacity of demographic variables - in this case, the fertility rate - to drive the economy towards the social optimum. To put it differently, when the Serendipity Theorem holds, if governments impose optimal fertility behaviour, then the rest of the economic variables will take automatically their optimal levels. Thus, Samuelson's result shows the power of demography as an instrument allowing for the decentralization of the long-run social optimum in an otherwise decentralized, perfectly competitive economy. ${ }^{4}$

Note, nevertheless, that the scope of Samuelson's results has been somewhat qualified by subsequent studies, such as the ones by Deardorff (1976), Michel and Pestieau (1993) and

\footnotetext{
${ }^{1}$ See Blackburn and Cipriani (2002), Doepke (2004), Galor and Moav (2005), de la Croix and Licandro (2007), and Cervellati and Sunde (2007).

${ }^{2}$ That assumption is clearly strong. We shall discuss that issue in more details below.

${ }^{3}$ In other words, imposing the optimum fertility makes the competitive economy converge towards the most golden rule steady-state, provided this one exists, is unique and stable. Otherwise, the convergence would not occur, and the social optimum could not be decentralized by means of the fertility rate.

${ }^{4}$ Note that taking demographic variables as inputs does not necessarily legitimate an intervention by the State. It is the particular approach adopted by Samuelson that treats demographic variables as instruments of the government, allowing for the decentralization of the social optimum, by making the solutions of the optimization problems of the social planner and of the agents coincide (see below).
} 
Jaeger and Kuhle (2009). Whereas those studies cast an important light on the scope of the Serendipity Theorem, these concentrate mainly on the characterization of the economic environment under which Samuelson's result holds, without refining the demographic environment. More precisely, those studies all keep the standard Diamond-type OLG structure, with a given fertility rate, and a fixed length of life equal to two periods. However, given that the Serendipity Theorem is about demography, one may want to know whether that result is robust to a finer characterization of the demography within the model, taking into account, in particular, the other major demographic variable: the longevity of agents.

The goal of this paper is precisely to re-examine the Serendipity Theorem in the context of an OLG economy with risky lifetime. In that economy, the demography becomes twodimensional: the population size and age-structure depend not only on the fertility rate, but, also, on the survival rate. Does the Serendipity Theorem still hold in that more general context, and, if yes, under which conditions?

The present paper proposes to answer that question. For that purpose, we shall consider a two-period OLG economy with physical capital accumulation, which is close to the Diamondtype economy studied by Samuelson, but with a major difference: unlike in Diamond (where all agents have a life of two periods of equal length), in our setting only a fraction of each cohort survives to the second period of life. That economy is actually quite close to the one whose dynamics is studied in the first part of Chakraborty's (2004) paper. After examining whether the Serendipity Theorem still holds in a simple framework where agents surviving to the second period are retirees, we shall, in a second stage, introduce labour supply during the second period (firstly as an exogenous fraction of the old-age, and then as a fraction chosen by the agents), and consider its consequences for the Serendipity Theorem.

Throughout this paper, it is assumed, in the spirit of Samuelson, that fertility and longevity are taken as given by agents, but can be controled by the social planner without cost. Such an approach is non standard, and looks like an inversion with respect to common sense. Clearly, a natural way to think about demographic variables consists of treating these as outputs, which cannot be directly controlled, but which can only be influenced indirectly, through some actions affecting the fundamentals of the economy. Samuelson's Serendipity Theorem starts from the opposite view, which is also embraced by the present paper. Undoubtedly, Samuelson's inversion could be regarded as quite counterintuitive, and, as such, invites some comments at this early stage of our investigations.

A first thing to be stressed is that assuming that fertility and longevity are perfectly controlable by the social planner without any cost - and taken as given by agents - constitutes an obvious simplification, which is only made here for analytical convenience. As such, the present study should be regarded as only a starting point inviting further developments. ${ }^{5}$ It seems of course more realistic to assume that both agents and the social planner can affect fertility and mortality indirectly, through economic variables of various kinds, those economic variables being themselves largely influenced by the demography. Thus Samuelson's approach involves a significant simplification.

\footnotetext{
${ }^{5}$ On the optimal cohort growth rate under costly, endogenous fertility, see Abio et al (2004). Regarding optimal longevity, de la Croix and Ponthiere (2010) study the optimal accumulation of capital in the context of a costly longevity.
} 
Having stressed this, one should not exaggerate the simplification involved in Samuelson's inversion. Although we shall not try here to provide a complete discussion on that complex issue, it should be noted that it is not obvious at all that economic variables are more easy to control than demographic variables. One reason why one could argue in favour of Samuelson's approach is that demographic variables can, at least, be easily observed, contrary to most economic variables, which are to a large extent unobservable. Another reason why one may argue in favour of Samuelson's approach is related to the speed of intervention: trying to influence demographic variables through the fundamentals of the economy may take ages, whereas simple reglementations on, let us say, fertility can do the job quite quickly, which may be most useful if governments face, for instance, urgent environmental constraints.

Naturally, none of those arguments is decisive, but at least these suggest that it is not obvious that Samuelson's inversion takes necessarily the problem in the wrong direction. The direction he proposes is also worth being explored, as a natural companion to studies proposing an indirect control of demographic variables. Moreover, in any case, Samuelson's approach allows us to have a concrete idea of the power of demography, i.e. of its influence on the functioning of the economy, and of its capacity (or incapacity) to lead to long-run optimality. These are the reasons why we shall pursue Samuelson's approach here.

Finally, one should also mention another major difference of Samuelson's approach with respect to the rest of the literature. The Serendipity Theorem is about how a social optimum can be decentralized by means of demographic variables, and, as such, this is a result with a strong normative content, since this involves the definition of a social optimum. That normative nature of the Serendipity Theorem is worth being underlined here, since the present study shares that feature with Samuelson's work. Note that discussing the decentralization of a social optimum only makes sense provided the social optimum is regarded as a reasonable social objective. This requirement is, in general, strong, but becomes even stronger in the context of varying population sizes (i.e. what Parfit (1984) calls different numbers choices). ${ }^{6}$ This is the reason why we shall pay here particular attention to the definition of the social optimum, and to its treatment of numbers, while remaining in a Samuelson-type framework.

This paper is organized as follows. Section 2 presents the model. Section 3 characterizes the optimal fertility rate and survival rate, and re-examines the Serendipity Theorem. Section 4 introduces (exogenous) second-period labour, and discusses the Serendipity Theorem in that context. Section 5 considers the case of endogenous old-age labour. Concluding remarks are drawn in Section 6.

\section{The model}

Let us consider a standard, Diamond-type OLG economy with physical capital accumulation. All agents live a first period of life (of length normalized to 1) for sure, during which they supply their labour inelastically, and save some resources for their old days.

\footnotetext{
${ }^{6}$ On the difficulties raised by population ethics, see Blackorby et al. (2005).
} 
Each young adult has also $n$ children during the first period. Hence, the labour force, denoted by $L_{t}$, follows the dynamic law:

$$
L_{t+1}=L_{t} n
$$

where $n$ can be interpreted as the fertility rate.

However, unlike in Diamond (1965), not all agents survive to the second period: only a fraction $\pi$ of the young cohort reaches the old age. In other words, life expectancy at birth in that economy equals $1+\pi$. Hence the population at time $t$ can be written as

$$
\begin{aligned}
N_{t} & =L_{t}+\pi L_{t-1} \\
& =L_{t-1}(n+\pi)
\end{aligned}
$$

The production of an output $Y_{t}$ involves capital $K_{t}$ and labour $L_{t}$, according to the function

$$
Y_{t}=F\left(K_{t}, L_{t}\right)=\bar{F}\left(K_{t}, L_{t}\right)+(1-\delta) K_{t}
$$

where $\delta$ is the depreciation rate of capital. ${ }^{7}$ The production function $\bar{F}\left(K_{t}, L_{t}\right)$ is assumed to be homogeneous of degree one. Hence, the total production function $F\left(K_{t}, L_{t}\right)$ is also homogeneous of degree one, and the production process as a whole can be rewritten in intensive terms as

$$
y_{t}=f\left(k_{t}\right)
$$

where $k_{t}$ denotes the capital per worker, while we have $f^{\prime}(k)>0$ and $f^{\prime \prime}(k)<0 .{ }^{8}$

The resource constraint of the economy, stating that what is produced is either consumed or invested, is:

$$
F\left(K_{t}, L_{t}\right)=c_{t} L_{t}+d_{t} \pi L_{t-1}+K_{t+1}
$$

At the steady state, and in intensive terms, we have

$$
f(k)-n k=c+\pi \frac{d}{n}
$$

Individual preferences are assumed to be represented by a function having the expected utility form, with a temporal utility function $u($.$) increasing and concave in consumption.$ Hence, provided the utility of death is normalized to zero, individual expected lifetime utility takes the form $u(c)+\pi u(d)$ where $c$ and $d$ denote first- and second-period consumptions. We assume $u^{\prime}()>$.0 and $u^{\prime \prime}()<$.0 .

\section{Optimum fertility rate and survival rate}

In order to consider what the Serendipity Theorem becomes in the economy under study, we shall first consider the social planner's problem, and derive the optimum levels of the fertility rate $n$ and the survival rate $\pi$.

\footnotetext{
${ }^{7}$ That function is used by de la Croix and Michel (2002, p. 4).

${ }^{8}$ More precisely, $k$ denotes the capital per young agent. In Sections 4 and 5 , we will allow old agents to work, so that capital per young agent and capital per worker will then differ.
} 


\subsection{The planner's problem}

Consider how the fertility rate $n$ and survival rate $\pi$ can be chosen in such a way as to reach the best of the optima, that is, the optimum optimorum. The problem of the social planner consists of choosing consumptions $c, d$ and capital $k$, and demographic variables $n$ and $\pi$ in such a way as to maximize the social objective function, subject to the resource constraint of the economy.

Throughout this paper, we will follow Samuelson (1975) and adopt, as a social objective function, the average lifetime welfare at the steady-state. That particular social objective invites two comments. Firstly, as this is well-known in the population ethics literature (see Blackorby et al. 2005), taking the average welfare rather than, let us say, the total welfare is far from neutral for different numbers choices, since this reveals a concern for the quality of lives rather than the quantity of lives. We shall come back to the consequences of that particular social objective for the issue at stake. Secondly, our emphasis on the steady-state welfare leads to neglect the interests of generations living in the transition, and, as such, is an imperfect social objective. Nonetheless, given that, by definition of the steady-state, an infinitely large number of generations will enjoy the living conditions prevailing at the steady-state, that exclusive focus on the steady-state can be regarded as a reasonable proxy.

The problem of the social planner can be written as:

$$
\max _{c, d, k, n, \pi} u(c)+\pi u(d) \text { subject to }(1)
$$

An interior optimum $\left(c^{\star}, d^{\star}, k^{\star}, n^{\star}, \pi^{\star}\right)$ should satisfy the following FOCs: ${ }^{9}$

$$
\begin{aligned}
\frac{u^{\prime}\left(c^{\star}\right)}{u^{\prime}\left(d^{\star}\right)} & =n^{\star} \\
f^{\prime}\left(k^{\star}\right) & =n^{\star} \\
f\left(k^{\star}\right)-n^{\star} k^{\star} & =c^{\star}+\pi^{\star} \frac{d^{\star}}{n^{\star}} \\
\pi^{\star} \frac{d^{\star}}{\left(n^{\star}\right)^{2}} & =k^{\star} \\
u\left(d^{\star}\right)-d^{\star} u^{\prime}\left(d^{\star}\right) & =0
\end{aligned}
$$

Condition (2) describes the optimal distribution of resources among generations, while condition (3) describes the optimum capital accumulation pattern. Expression (4) is the feasibility constraint. Taken together, those three conditions characterize usually the optimal levels of $c, d$ and $k$ for given levels of $n$ and $\pi$. Condition (5) defines implicitly the optimum fertility rate: as stressed by Jaeger and Kuhle (2009), it explicitates the trade-off between the negative capital widening effect (i.e. $k$ ) and the positive intergenerational transfer effect (i.e. $\pi \frac{d}{n^{2}}$ ), whose size depends positively on the survival rate $\pi$. Those two effects of fertility

\footnotetext{
${ }^{9}$ In the spirit of Samuelson's work, we will focus mainly here on conditions characterizing an interior optimum. However, we will discuss the plausibility of a corner optimum survival rate below. Note also that we assume, like Samuelson, that the social optimum is unique, that is, that only one set of variables satisfy the above FOCs. This guarantees that our optimum is necessarily a global optimum.
} 
are playing in opposite directions, so that there seems to be some intuitive support for the existence of an interior optimum fertility rate. Given that the extra-value of the present study lies in the introduction of mortality, we will, for the sake of presentation, assume, in the rest of this paper, that the optimum fertility rate is an interior optimum. ${ }^{10}$

Let us now pay more attention to condition (6), which characterizes the interior optimum survival rate. Condition (6) states that the marginal welfare gain from a higher survival prospect, $u(d)$, should be equal to the marginal cost of a higher survival prospect, which is given by $d u^{\prime}(d)$. That condition is necessary for the existence of an interior optimum survival rate.

Remark: Equation (6) can be rewritten as

$$
\frac{u^{\prime}(d)}{u(d)}=\frac{1}{d}
$$

where the LHS is the fear of ruin, a measure of risk-aversion.

Under which circumstances is condition (6) satisfied? Whether $u(d)$ is inferior, equal or superior to $d u^{\prime}(d)$ depends on several properties of the economy. A first determinant consists of the shape of the temporal utility function $u($.$) , and, in particular, of its intercept. { }^{11}$ If $u(0)>0$ or $u(0)=0$, then, given the concavity of $u($.$) , it is always the case that u(d)$ exceeds $d u^{\prime}(d)$ for $d>0$, so that the optimum survival rate $\pi^{*}$ equals 1 . On the contrary, if $u(0)<0$, then we may have an interior optimum $\pi^{*}$ or not, depending on the level of second-period consumption at the social optimum. This leads us to a second determinant of $\pi^{*}$ : the production capacity of the economy, as captured by the production function $f\left(k_{t}\right)$. If the production technique is efficient, optimum output and consumption levels are high, so that it is likely that $\pi^{*}$ is strictly positive, despite a negative intercept. On the contrary, if the production technique is not efficient, optimum second-period consumption is low, and then, under $u(0)<0$, we have $\pi^{*}$ equal to $0 .{ }^{12}$

One can illustrate that discussion on the interiority of $\pi^{*}$ by means of the concept of value of a statistical life.

Remark: The value of a statistical life (VSL), defined as the shadow price of a reduction of the risk of death per unit of risk, can be rewritten as

$$
\frac{\frac{\partial U}{\partial \pi}}{\frac{\partial U}{\partial d}}=\frac{u(d)-d u^{\prime}(d)}{u^{\prime}(d)}
$$

which is positive only provided $u(d)-d u^{\prime}(d)>0$.

\footnotetext{
${ }^{10}$ Readers interested in the interiority or non-interiority of the optimum fertility rate can refer to Deardorff (1976), Michel and Pestieau (1993) and Abio (2003).

${ }^{11}$ To illustrate the crucial role played by the shape of $u(\cdot)$, the Appendix concentrates on the case of a CIES utility function with an intercept.

${ }^{12}$ In that case, the low production capacity, by restraining consumption below a level making a life-period enjoyable, makes a short life better than a long life from the perspective of total lifetime welfare.
} 
If the value of a statistical life at the social optimum is positive, the condition for an interior optimum survival rate is unlikely to be satisfied, as we have

$$
u(d)-d u^{\prime}(d)>0
$$

Thus, if the VSL is positive at the social optimum, the optimum survival rate is not an interior solution, but a corner solution, equal to $\pi^{*}=1$. On the contrary, if, at the social optimum, the VSL is negative, we have

$$
u(d)-d u^{\prime}(d)<0
$$

so that the condition (6) is never satisfied, and we have the other corner solution: $\pi^{\star}=0$.

It is thus only in the special case of a zero VSL at the optimum that condition (6) holds.

In sum, whether condition (6), (7) or (8) prevails depends on the shape of individual temporal utility functions, and on the level of old-age consumption at the social optimum. In general, it is widely acknowledged in the empirical literature on VSL estimates that the VSL is positive and is increasing in the level of wealth. Hence, if, at the laissez-faire, the VSL is positive (which is the case), and if the social optimum involves a higher old-age consumption than the laissez-faire (which is also plausible), the VSL must also be positive at the social optimum (as the VSL is increasing in $d$ ), implying that $u(d)-d u^{\prime}(d)>0$ holds, so that the optimum survival rate is, under those assumptions, a corner solution.

Therefore, in the rest of this paper, we shall take that possibility into account, and derive our results for an interior optimum survival rate $0<\pi^{*}<1$ and a corner solution $\pi^{*}=1$. On the contrary, we shall leave aside the - unrealistic - case where $\pi^{*}=0 .{ }^{13}$

Finally, it should be noted that the above first-order conditions are necessary, but not sufficient, for $\left(c^{\star}, d^{\star}, k^{\star}, n^{\star}, \pi^{\star}\right)$ to be a maximum. Actually, a sufficient condition for a maximum is that the first-order derivatives are equal to zero, and that the Hessian matrix of this optimization problem is negative definite. The second-order conditions are: ${ }^{14}$

$$
\begin{aligned}
\frac{\left(\pi^{\star}\right)^{2} u^{\prime \prime}\left(c^{\star}\right)}{n^{\star}}+n^{\star} \pi^{\star} u^{\prime \prime}\left(d^{\star}\right) & <0 \\
n^{\star} \pi^{\star} u^{\prime}\left(d^{\star}\right) f^{\prime \prime}\left(k^{\star}\right)\left[\pi^{\star} u^{\prime \prime}\left(c^{\star}\right)+\left(n^{\star}\right)^{2} u^{\prime \prime}\left(d^{\star}\right)\right] & >0 \\
\pi^{\star} u^{\prime}\left(d^{\star}\right)\left[\left(n^{\star}\right)^{2} \pi^{\star}\left[u^{\prime}\left(d^{\star}\right)\right]^{2} f^{\prime \prime}\left(k^{\star}\right)+\left(n^{\star}\right)^{2} u^{\prime}\left(d^{\star}\right)\left[\pi^{\star}\left(n^{\star}+2 k^{\star} f^{\prime \prime}\left(k^{\star}\right)\right) u^{\prime \prime}\left(c^{\star}\right)\right]\right] & \\
-\pi^{\star} u^{\prime}\left(d^{\star}\right)\left(\left(n^{\star}\right)^{3}+2 d^{\star} \pi^{\star} f^{\prime \prime}\left(k^{\star}\right)\right) u^{\prime \prime}\left(d^{\star}\right) & <0 \\
-\left(d^{\star}\right)^{2}\left(n^{\star}\right)^{3} \pi^{\star}\left[u^{\prime}\left(d^{\star}\right)\right]^{2}\left[u^{\prime}\left(d^{\star}\right) f^{\prime \prime}\left(k^{\star}\right)+\left(n^{\star}+2 k^{\star} f^{\prime \prime}\left(k^{\star}\right)\right) u^{\prime \prime}\left(c^{\star}\right)\right] u^{\prime \prime}\left(d^{\star}\right) & >0
\end{aligned}
$$

Conditions (9) and (10) are, by the concavity of the temporal utility $u(\cdot)$ and of the production function $f(\cdot)$, always verified. However, the same is not true for conditions (11) and (12). It is actually quite difficult to see whether those two conditions are likely to be satisfied. To discuss this, it is necessary to impose particular functional forms for the temporal utility $u(\cdot)$ and for the production function $f(\cdot) \cdot{ }^{15}$

\footnotetext{
${ }^{13}$ Actually, that case seems implausible for advanced economies with large production capacities.

${ }^{14}$ See the Appendix for the derivation.

${ }^{15}$ In the Appendix, we derive the FOCs and SOCs in the special case of a CIES utility function and a Cobb-Douglas production function, and discuss the cases under which those conditions are satisfied.
} 


\subsection{The Serendipity Theorem}

Let us now consider a competitive economy and assume that a steady-state equilibrium exists, and is unique and stable. Factors are paid at their marginal productivity:

$$
\begin{aligned}
& w=f(k)-k f^{\prime}(k) \\
& R=f^{\prime}(k)
\end{aligned}
$$

where $w$ is the wage, while $R$ is the return on capital.

For the sake of space constraints, the existence and uniqueness of a stationary equilibrium are not discussed here formally. However, provided agents are expected utility maximizers and provided there is a perfect annuity market with actuarially fair returns, it can be shown that an equilibrium exists under the assumptions identified by de la Croix and Michel (2002, chapter 1): $u^{\prime}(c)>0, u^{\prime \prime}(c)<0$, and $\lim _{c_{t} \rightarrow 0} u^{\prime}(c)=+\infty$, as well as, for all $k>0$, $f(k)>0, f^{\prime}(k)>0$ and $f^{\prime \prime}(k)<0$. Moreover, the condition guaranteeing the uniqueness of a stationary equilibrium in de la Croix and Michel (2002, p. 34) can also be easily extended to the case of risky lifetime. Note, however, that the risk of death has an ambiguous effect on the uniqueness condition, as it affects both the time horizon and the savings return (see Chakraborty, 2004).

The problem faced by a young agent is to choose his optimal savings subject to his budget constraint. Following the literature, we assume that there exists a perfect annuity market here, which yields an actuarially fair return on savings. ${ }^{16}$ Hence, under that assumption, the second period consumption is

$$
d=\frac{R s}{\pi}
$$

where $s$ denotes individual savings. Under $w=c+s$, the lifetime budget constraint of the agent can be written as

$$
w=c+\pi \frac{d}{R}
$$

Under those assumptions, the problem of a young agent can thus be written as

$$
\max _{c, d} u(c)+\pi u(d) \text { subject to } w=c+\pi \frac{d}{R}
$$

It should be stressed here that the objective function of the agent, which consists of his expected lifetime welfare, coincides formally with the average lifetime utility when evaluated ex post, which consists of nothing else than the social planner's objective. Thus, even though the optimization problems of the social planner and the agent are fundamentally different, there is an interesting similarity in the functional forms of the objective functions, thanks to the coincidence between the ex post average lifetime welfare (i.e. the objective of the planner) and the ex ante expected lifetime welfare (i.e. the objective of the agent).

\footnotetext{
${ }^{16}$ That assumption, which is most common in the literature (see Chakraborty 2004), involves a strong simplification, since annuity markets are largely underdeveloped in the real world (see Brown 2007). Note, however, that the Serendipity Theorem concerns a perfectly competitive economy, so that assuming perfect annuity markets seems to be the most natural assumption for the purpose at hand.
} 
From the FOCs of the agent's problem, we have

$$
\frac{u^{\prime}(c)}{u^{\prime}(d)}=R
$$

Finally, in equilibrium, capital equals savings:

$$
n k=s=w-c
$$

For given $(n, \pi)$, a stationary competitive equilibrium is a vector $(\tilde{w}, \tilde{R}, \tilde{c}, \tilde{d}, \tilde{k})$ satisfying (13)-(17).

Assume that $n$ is at its optimum value $n^{\star}$, i.e. (5) holds. Then, the market conditions (13)-(17) would coincide with the optimal conditions (2)-(3)-(4). If we abstracts from the survival rate, this result coincides with the standard Serendipity Theorem: imposing the optimum fertility rate suffices to bring the economy at the optimum steady-state.

However, the condition (6) for an interior optimum survival rate - or conditions (7) and (8) for a corner optimum survival rate - does not follow automatically from individual behaviour under the optimum fertility rate, and may remain non-satisfied if only the optimum fertility rate is imposed.

Nevertheless, if the social planner can impose both the optimum fertility rate and the optimum survival rate (assumed to be strictly positive), then, it is straightforward to see from the above FOCs that all the other variables of the economy take their optimum values. The following proposition summarizes the results. ${ }^{17}$

Proposition 1 Suppose that there exists a unique and stable steady-state equilibrium with a positive capital $k>0$.

Assume that Conditions (2)-(5), (6), and (9)-(12) hold, then, if the government imposes $n^{\star}$ and $\pi^{\star}$, the economy will converge towards the optimum steady-state, which involves $0<n^{\star}<+\infty$ and $0<\pi^{\star}<1$.

Assume that Conditions (2)-(5), (7), and (9)-(11) hold, then, if the government imposes $n^{\star}$ and $\pi^{\star}$, the economy will converge towards the optimum steady-state, which involves $0<n^{\star}<+\infty$ and $\pi^{\star}=1$.

Therefore, the introduction of survival rates in the OLG framework does not invalidate the Serendipity Theorem. ${ }^{18}$ However, it is true that, in that extended framework, a government

\footnotetext{
${ }^{17}$ Note that, in the case where the optimum survival rate $\pi^{\star}$ is zero, this Extended Serendipity Theorem does not hold. Indeed, if the social planner imposes $\pi^{\star}=0$ to individuals, these will not save any more. As a consequence, we have, at the steady-state, $k=0$, whereas the Serendipity Theorem focuses on steady-states with a strictly positive capital level.

${ }^{18}$ Note also that, if agents could, at the laissez-faire, affect their survival probability (e.g. through health spending), then there may be a difference between the laissez-faire and the social optimum, as agents may ignore the effect of their health investment on the return of their savings, and choose a non-optimal level of health investment (see Becker and Philipson, 1998). This possibility does not arise here, where agents take the demographic parameters $n$ and $\pi$ as given.
} 
can only drive the economy towards its optimum steady-state provided it imposes both the optimum fertility rate and the optimum survival rate. In general (i.e. provided the optimum old-age consumption is sufficiently large), the optimum $\pi$ is equal to 1 , so that the Serendipity Theorem requires here to impose maximal and riskless longevity for all, in such a way as to reach the optimum long-run equilibrium.

At this stage, it should be stressed that the above Extended Serendipity Theorem relies on a particular set of assumptions. Let us provide here a brief, preliminary discussion of the robustness of the Extended Serendipity Theorem, to be followed by further developments in the next sections of this paper.

A first, important issue to be discussed regarding the robustness of our results concerns the modelling of longevity. Actually, it should be stressed that the particular way in which mortality is introduced in our model does not seem determinant for the validity of the Extended Serendipity Theorem. More precisely, there exist two main ways to introduce mortality in a two-period OLG model, and our result is robust to adopting the other modelling of longevity. That alternative modelling would not involve risk, but, rather, would consist of a second period of length $h$ lived by all agents ( $h$ being between 0 and 1). ${ }^{19}$ An obvious cost of that alternative modelling is that the absence of risk makes the model less realistic, but, at the same time, there is a gain from the fact that we do not need there to assume perfect annuity markets, precisely because risk has disappeared. It is straightforward to check that, under that alternative setting, the FOCs of the social planner's problem and of the agent's problem would remain exactly as they are in our framework (except, of course, that $\pi$ is replaced by $h$ ). As a consequence, the Extended Serendipity Theorem would still hold under that alternative modelling of longevity. ${ }^{20}$

While the Extended Serendipity Theorem is robust to how longevity is modelled, the same cannot be said for several other aspects of the model. The Extended Serendipity Theorem consists, in the same way as Samuelson's theorem, of a happy coincidence result, which relies on some assumptions allowing for the formal similarity of the FOCs describing the solutions of the social planner and the agent's problems. An important assumption consists of the formal similarity between the objective functions of the social planner and of the agent, as mentioned above. If, for instance, the social planner was a critical-level utilitarian planner, then this would introduce a difference between the two functional forms at stake, and would make the happy coincidence disappear. ${ }^{21}$ Alternatively, if agents were not expected utility maximizers, but had different preferences, the formal similarity between the two objective functions would also disappear, and the Extended Serendipity Theorem may also collapse. Moreover, the assumption of a perfect annuity market yielding an actuarially fair return,

\footnotetext{
${ }^{19}$ In that alternative framework, life expectancy is equal to $1+h$, against $1+\pi$ in the present setting, but the difference is that survival curves would then be perfectly rectangular (see Jouvet et al. 2010).

${ }^{20}$ Note also that there exists a third way to introduce longevity in a two-period setting: this consists of making agents face a probability $\pi$ to enjoy a second period of length $h$, which yields a life expectancy equal to $1+\pi h$ (see Ponthiere 2009). Under that alternative framework, the Extended Serendipity Theorem would still hold provided perfect annuity markets and average utilitarianism are assumed.

${ }^{21}$ Under critical-level utilitarianism, the social objective function is not an average of individual utilities, but a sum of individual utilities net of a critical welfare level (which, by definition, makes a life neutral from the perspective of social welfare).
} 
which amounts to assume a perfect division of the risk of death on the whole cohort, is also a most convenient way to minimize the differences between the social planner's problem and the agent's problem, which yields, in fine, the happy coincidence. Relaxing that assumption may not preserve the Extended Serendipity Theorem. Note, however, that this point should not surprise us, since the assumption of perfectly competitive markets was already playing a crucial role in Samuelson's initial result. This remains true in our extended framework.

Those remarks suffice to highlight that, exactly as Samuelson's initial result, the Extended Serendipity Theorem is dependent on a conjunction of hypotheses. In Samuelson's model, the survival probability was fixed to 1 and not chosen by the planner. As a consequence, controlling for the fertility rate was similar to controlling for the whole demography. On the contrary, in our extended framework, both the fertility rate and survival rate need to be controlled to decentralize the social optimum. That result could have been expected, since, here again, controlling for both $n$ and $\pi$ coincides with a control of the whole demography. Note, however, that the above observations suggest that the extension is not as automatic as it may appear at first glance, since this requires particular assumptions allowing for the happy coincidence. In the rest of this paper, we shall now explore the robustness of the Extended Serendipity Theorem further, by refining other aspects of our model.

\section{Exogenous old-age labour}

Whereas Section 3 showed that the introduction of mortality within an OLG economy does not invalidate, but only leads to a re-qualification, of the Serendipity Theorem, it may be argued that the introduction of mortality was, in the above framework, not capturing the overall effect of ageing on the economy. The reason why one may remain unsatisfied is that the above model treats all survivors to the second period as retirees.

Actually, that assumption is not fully realistic, because one may expect that, as the life expectancy grows, the working time grows also. But besides the issue of realism, one may also argue that the precise way in which mortality was introduced in Section 3, by leaving the labour supply unchanged, may not be neutral as far as the validity of the Extended Serendipity Theorem is concerned. Thus it is worth exploring here whether our results are robust to a more general modelling where the survival rate $\pi$ affects the total labour supply through its impact on old-age labour. ${ }^{22}$

Hence, this section aims at complementing the basic model developed above by a more realistic one, where agents surviving to the second period can also take part in the production process. This will be the opportunity to evaluate the robustness of the Extended Serendipity Theorem to a more general modelling of the lifecycle. Undoubtedly, introducing old-age labour adds additional connections between fertility and mortality, as we shall now see.

\footnotetext{
${ }^{22}$ Actually, mortality, as introduced in Section 3, does not affect the proportion of current savers with respect to future workers, which is a key ratio explaining why the demography matters in the theory of optimal capital accumulation. We explore here what happens when $\pi$ affects that ratio.
} 
Let us now assume that the surviving old agents work an exogenous fraction $\lambda$ of the second period, with $0<\lambda<1$. $^{23}$ Hence the total labour force becomes

$$
L_{t}+\lambda \pi L_{t-1}=L_{t}\left(1+\frac{\lambda \pi}{n}\right)
$$

Production still involves capital and labour as before. For simplicity, it is assumed that old workers have the same productivity as young ones (i.e. they compensate exactly their old age by their additional experience)

$$
Y_{t}=F\left(K_{t}, L_{t}\left(1+\frac{\lambda \pi}{n}\right)\right)
$$

Hence, under CRS, production can be written, in intensive terms, as

$$
y_{t}=F\left(k_{t}, 1+\frac{\lambda \pi}{n}\right)
$$

where $y_{t}$ is the output per young adult, and $k_{t}$ is the capital per young adult.

The resource constraint is derived as follows. We have

$$
K_{t+1}=F\left(K_{t}, L_{t}\left(1+\frac{\lambda \pi}{n}\right)\right)-L_{t} c_{t}-\pi L_{t-1} d_{t}
$$

Hence we have, at the steady-state

$$
F\left(k, 1+\frac{\lambda \pi}{n}\right)-n k=c+\pi \frac{d}{n}
$$

\subsection{The social planner's problem}

Under that alternative framework, the problem of the social planner becomes

$$
\max _{c, d, k, n, \pi} u(c)+\pi u(d) \text { subject to }(18)
$$

Under the FOCs, we have, at an interior optimum,

$$
\begin{aligned}
\frac{u^{\prime}\left(c^{\star}\right)}{u^{\prime}\left(d^{\star}\right)} & =n^{\star} \\
F_{k}\left(k^{\star}, 1+\frac{\lambda \pi^{\star}}{n^{\star}}\right) & =n^{\star} \\
F\left(k^{\star}, 1+\frac{\lambda \pi^{\star}}{n^{\star}}\right)-n^{\star} k^{\star} & =c^{\star}+\pi^{\star} \frac{d^{\star}}{n^{\star}} \\
-k^{\star}+\pi^{\star} \frac{d^{\star}}{\left(n^{\star}\right)^{2}}-F_{L}\left(k^{\star}, 1+\frac{\lambda \pi^{\star}}{n^{\star}}\right) \frac{\lambda \pi^{\star}}{\left(n^{\star}\right)^{2}} & =0 \\
u\left(d^{\star}\right)-d^{\star} u^{\prime}\left(d^{\star}\right)+F_{L}\left(k^{\star}, 1+\frac{\lambda \pi^{\star}}{n^{\star}}\right) \lambda u^{\prime}\left(d^{\star}\right) & =0
\end{aligned}
$$

\footnotetext{
${ }^{23}$ Note that there may exist other ways to introduce old-age labour in the present framework. Here we adopted the most convenient formalization.
} 
In comparison with the basic model, the second condition is different, as the marginal productivity of capital depends on the survival rate, unlike what used to prevail before. Similarly, the condition (21) - the feasibility constraint - is also affected by old-age workers: the survival rate has here a positive effect on the feasible set.

There is another difference with the basic model [i.e. condition (5)]: condition (22), which characterizes the optimum fertility rate, contains an additional term, the third one, which captures the negative effect of $n$ on the output per young worker $y_{t}{ }^{24}$ The two other effects are the same as in the standard model. Thus, if we compare the optimum interior fertility under old-age labour (i.e. $\lambda>0$ ), we see that the optimum $n$ is likely to be smaller than without old-age labour (i.e. $\lambda=0$ ).

Condition (23) characterizes the interior optimum survival rate. Here again, there is an additional term in comparison with condition (6), which captures the positive effect of a higher survival rate on the labour force, and, hence, on the output per young worker. That additional, positive term is likely to reinforce the likelihood of a corner solution for optimum $\pi$, i.e. for $\pi^{\star}=1$ :

$$
u(d)-d u^{\prime}(d)+F_{L}\left(k, 1+\frac{\lambda \pi}{n}\right) \lambda u^{\prime}(d)>0
$$

Hence, the positive influence of the survival rate on the feasibility set makes the condition for the corner solution $\pi^{\star}=0$, which is

$$
u(d)-d u^{\prime}(d)+F_{L}\left(k, 1+\frac{\lambda \pi}{n}\right) \lambda u^{\prime}(d)<0
$$

even less plausible than under no old-age labour (i.e. $\lambda=0$ ).

Finally, the sufficient conditions for $\left(c^{\star}, d^{\star}, k^{\star}, n^{\star}, \pi^{\star}\right)$ to be a maximum are now: ${ }^{25}$

$$
\begin{aligned}
\frac{\left(\pi^{\star}\right)^{2} u^{\prime \prime}\left(c^{\star}\right)}{n^{\star}}+n^{\star} \pi^{\star} u^{\prime \prime}\left(d^{\star}\right) & <0 \\
n^{\star} \pi^{\star} u^{\prime}\left(d^{\star}\right) F_{K K}\left[\pi^{\star} u^{\prime \prime}\left(c^{\star}\right)+\left(n^{\star}\right)^{2} u^{\prime \prime}\left(d^{\star}\right)\right] & >0 \\
n^{\star} \pi^{\star} u^{\prime}\left(d^{\star}\right)\left[\left(\pi^{\star}\right)^{2} F_{K L}^{2} u^{\prime}\left(d^{\star}\right) \varphi-F_{K K}\left(n^{\star} F_{L}^{2} u^{\prime \prime}\left(c^{\star}\right) u^{\prime \prime}\left(d^{\star}\right)\right)\right] & \\
-n^{\star} \pi^{\star} u^{\prime}\left(d^{\star}\right) F_{K K}\left(\left(\pi^{\star}\right)^{2} F_{L L} u^{\prime}\left(d^{\star}\right) \varphi\right) & <0 \\
\pi^{\star}\left[u^{\prime}\left(d^{\star}\right)\right]^{2}\left[\Gamma+\left(n^{\star}\right)^{3} \Delta+\left(\pi^{\star}\right)^{2} F_{K L}^{2} \Theta-\left(\pi^{\star}\right)^{2} F_{L L} \Lambda\right] & >0
\end{aligned}
$$

\footnotetext{
${ }^{24}$ That additional negative effect tends to counteract the standard intergenerational transfer effect mentioned in Section 3. Indeed condition (22) can be rewritten as

$$
-k^{\star}+\frac{\pi^{\star}}{\left(n^{\star}\right)^{2}}\left(d^{\star}-\lambda F_{L}\left(k^{\star}, 1+\frac{\lambda \pi^{\star}}{n^{\star}}\right)\right)=0
$$

The standard intergenerational transfer effect (i.e. the second term) is here reduced by the fact that old agents do not only consume, but also produce (under $\lambda>0$ ), as shown by the second term in brackets.

${ }^{25}$ See the Appendix for the derivation.
} 
with

$$
\begin{aligned}
\varphi \equiv & \pi^{\star} u^{\prime \prime}\left(c^{\star}\right)+\left(n^{\star}\right)^{2} u^{\prime \prime}\left(d^{\star}\right) \\
\Gamma \equiv & 2\left(n^{\star}\right)^{2} \pi^{\star} F_{K L}\left(-\lambda F_{L}^{2} u^{\prime \prime}\left(c^{\star}\right) u^{\prime \prime}\left(d^{\star}\right)+\left(n^{\star}\right)^{2} F_{L} u^{\prime}\left(d^{\star}\right) u^{\prime \prime}\left(d^{\star}\right)\right), \\
\Delta \equiv & -F_{L}^{2}\left(\left(n^{\star}+2 k^{\star} F_{K K}\right) u^{\prime \prime}\left(c^{\star}\right)+F_{K K} u^{\prime}\left(d^{\star}\right)\right) u^{\prime \prime}\left(d^{\star}\right) \\
& +\pi^{\star}\left(\pi^{\star}\left(n^{\star}+2 k^{\star} F_{K K}\right) u^{\prime \prime}\left(c^{\star}\right)+\left(n^{\star}\right)^{3} u^{\prime \prime}\left(d^{\star}\right),\right. \\
\Theta \equiv & \left(n^{\star}\right)^{2} \pi^{\star}\left(u^{\prime}\left(d^{\star}\right)\right)^{2}+u^{\prime \prime}\left(c^{\star}\right)\left(2 k^{\star}\left(n^{\star}\right)^{2} \pi^{\star} u^{\prime}\left(d^{\star}\right)+\lambda^{2}\left(-F_{L}^{2} u^{\prime \prime}\left(d^{\star}\right)\right)\right), \\
\Lambda \equiv & u^{\prime \prime}\left(c^{\star}\right)\left[\left(n^{\star}\right)^{2} \pi^{\star}\left(n^{\star}+2 k^{\star} F_{K K}\right) u^{\prime}\left(d^{\star}\right)+\lambda^{2} F_{K K}\left(-F_{L}^{2} u^{\prime \prime}\left(d^{\star}\right)\right)\right], \\
& +n^{2}\left(\left(n^{\star}\right)^{3} u^{\prime}\left(d^{\star}\right) u^{\prime \prime}\left(d^{\star}\right)+F_{K K}\left(\pi^{\star}\left(u^{\prime}\left(d^{\star}\right)\right)^{2}+2\left(k^{\star}\left(n^{\star}\right)^{2}+\lambda F_{L}\right) u^{\prime}\left(d^{\star}\right) u^{\prime \prime}\left(d^{\star}\right)\right)\right) .
\end{aligned}
$$

Conditions (26) and (27) are identical to conditions (9) and (10) of the model without old-age labour. As in the basic model, it is straightforward to see that those conditions are always satisfied given the concavity of the temporal utility function and of the production function. However, conditions (28) and (29) differ from (11) and (12). Moreover, those two conditions are not necessarily satisfied.

\subsection{The Serendipity Theorem}

We now look at the competitive steady state assuming that it is unique and stable. As before, factors are paid at their marginal productivities:

$$
\begin{aligned}
w & =\left(F\left(k, 1+\frac{\lambda \pi}{n}\right)-k F_{k}\left(k, 1+\frac{\lambda \pi}{n}\right)\right) \frac{n}{n+\lambda \pi} \\
R & =F_{k}\left(k, 1+\frac{\lambda \pi}{n}\right)
\end{aligned}
$$

We can now consider the problem faced by a young agent, who chooses his optimal savings subject to his budget constraint. Under a perfect annuity market with an actuarially fair return on savings, the second-period consumption is, under an (exogenous) second-period labour $\lambda$, equal to

$$
d=\frac{R s}{\pi}+\lambda w
$$

Under $w=c+s$, the budget constraint of the agent can be written as

$$
w=\left(c+\pi \frac{d}{R}\right)\left(\frac{R}{R+\lambda \pi}\right)
$$

The problem of a young agent can thus be written as

$$
\max _{c, d} u(c)+\pi u(d)
$$

subject to the above budget constraint. From the FOCs, we have the same equation as before, i.e. Equation (16). Finally, in equilibrium, capital equals savings, i.e. (17) holds. 
For given $(n, \pi)$, a stationary competitive equilibrium is a vector $(\tilde{w}, \tilde{R}, \tilde{c}, \tilde{d}, \tilde{k})$ satisfying (16), (17), and (30)-(32).

Assume that $n$ is at its optimum value $n^{\star}$, i.e. (22) holds. Then, the market conditions (16), (17), and (30)-(32) would coincide with the optimal conditions (19)-(21). Thus, once old-age labour is introduced $(\lambda>0)$, and if we abstract from the choice of the optimal survival rate, the Serendipity Theorem still holds: imposing the optimum fertility rate suffices to bring the economy at the optimum steady-state.

Once we consider the choice of the optimal survival rate, it is clear, here again, that the condition (23) for an interior optimum survival rate - or the conditions (24) and (25) for a corner optimum survival rate - do not follow automatically from individual behaviour under the optimum fertility rate.

Nevertheless, if the social planner can impose both the optimum fertility rate and the optimum survival rate (still assumed to be strictly positive), then, here again, all the other variables of the economy take also their optimum values. The only difference with respect to the basic model consists of the levels of those optimum levels. The following proposition summarizes the results.

Proposition 2 Suppose that there exists a unique and stable steady-state equilibrium with a positive capital $k>0$.

Assume that Conditions (19)-(22), (23), and (26)-(29) hold, then, if the government imposes $n^{\star}$ and $\pi^{\star}$, the economy will converge towards the optimum steady-state, which involves $0<n^{\star}<+\infty$ and $0<\pi^{\star}<1$.

Assume that Conditions (19)-(22), (24) and (26)-(28), then, if the government imposes $n^{\star}$ and $\pi^{\star}$, the economy will converge towards the optimum steady-state, which involves $0<n^{\star}<+\infty$ and $\pi^{\star}=1$.

Therefore, the introduction of old-age labour does not invalidate the Extended Serendipity Theorem. Naturally, it is true that, under the possibility of second-period work, the incentive to save is likely to be reduced, so that the mere existence of a steady-state equilibrium with a strictly positive capital level becomes here a stronger assumption. However, provided the existence, uniqueness and stability of the stationary equilibrium still hold, Proposition 2 tells us that, if the government can impose the optimum levels of $n$ and $\pi$ - which differ from their levels in the basic model - then it is still guaranteed that the economy will converge towards the optimum steady-state. In sum, the introduction of old-age labour only changes the level of the target for the fertility rate $n$ - and, possibly, the one for $\pi$ - but not the possibility to reach the social optimum by merely imposing the optimum pair $\left(n^{\star}, \pi^{\star}\right)$.

\section{Endogenous old-age labour}

Finally, let us conclude our re-examination of the Serendipity Theorem by considering the case where old-age labour is chosen by agents when being young, and where there exists some 
disutility from old-age labour. While assuming that agents choose the length of their working time is not fully realistic (strong legislative limitations still prevail in some countries), it may be worth, nonetheless, to develop that alternative case, to see how robust our results are to the exogeneity of old-age labour supply.

Assume now that young adults choose their old-age working time by maximizing their expected lifetime welfare

$$
u\left(c_{t}\right)+\pi u\left(d_{t+1}\right)-\pi v\left(\lambda_{t+1}\right)
$$

where $v(\cdot)$ denotes the disutility of second-period labour. ${ }^{26}$ We assume that the disutility of old-age labour is a strictly increasing, convex function. We also have $\lim _{\lambda_{t+1} \rightarrow 0} v^{\prime}\left(\lambda_{t+1}\right)=0$ and $\lim _{\lambda_{t+1} \rightarrow 1} v^{\prime}\left(\lambda_{t+1}\right)=+\infty$.

Note that this modelling of the retirement decision implies that agents choose their retirement age at the same time as they choose their savings, that is, at the beginning of their life. It may be argued that, in real life, agents choose their retirement age at a higher age, but here we prefer assuming, for analytical simplicity, that each agent has a unique decision node, so that his whole life-plan (savings and retirement age) is necessarily consistent. ${ }^{27}$

As above, production still involves capital and labour. For simplicity, it is still assumed that old workers have the same productivity as young ones. Hence total output is

$$
Y_{t}=F\left(K_{t}, L_{t}\left(1+\frac{\lambda_{t} \pi}{n}\right)\right)
$$

where $L_{t}\left(1+\frac{\lambda_{t} \pi}{n}\right)$ is the total labour force. The only difference with respect to the previous section is that the old-age labour time $\lambda_{t}$ is now endogenous. Under CRS, production can be written, in intensive terms, as

$$
y_{t}=F\left(k_{t}, 1+\frac{\lambda_{t} \pi}{n}\right)
$$

where $y_{t}$ is the output per young adult, and $k_{t}$ is the capital per young adult.

\subsection{The social planner's problem}

Under that alternative framework, the problem of the social planner becomes

$$
\max _{c, d, k, \lambda, n, \pi} u(c)+\pi u(d)-\pi v(\lambda) \text { subject to }
$$

\footnotetext{
${ }^{26}$ That modeling is close to the one in $\mathrm{Hu}$ (1979) and de la Croix et al (2004), but for risky longevity.

${ }^{27}$ Note, however, that the agent's objective function is, in our model, additive over time, so that the decision concerning the retirement age is necessarily time-consistent.
} 
Under the FOCs, we have, at an interior optimum:

$$
\begin{aligned}
\frac{u^{\prime}\left(c^{\star}\right)}{u^{\prime}\left(d^{\star}\right)} & =n^{\star} \\
F_{k}\left(k^{\star}, 1+\frac{\lambda^{\star} \pi^{\star}}{n^{\star}}\right) & =n^{\star} \\
u^{\prime}\left(c^{\star}\right) F_{L}\left(k^{\star}, 1+\frac{\lambda^{\star} \pi^{\star}}{n^{\star}}\right) \frac{1}{n^{\star}} & =v^{\prime}\left(\lambda^{\star}\right) \\
F\left(k^{\star}, 1+\frac{\lambda^{\star} \pi^{\star}}{n^{\star}}\right)-n^{\star} k^{\star} & =c^{\star}+\pi^{\star} \frac{d^{\star}}{n^{\star}} \\
-k^{\star}+\pi^{\star} \frac{d^{\star}}{\left(n^{\star}\right)^{2}}-F_{L}\left(k^{\star}, 1+\frac{\lambda^{\star} \pi^{\star}}{n^{\star}}\right) \frac{\lambda^{\star} \pi^{\star}}{\left(n^{\star}\right)^{2}} & =0 \\
u\left(d^{\star}\right)-d^{\star} u^{\prime}\left(d^{\star}\right)+F_{L}\left(k^{\star}, 1+\frac{\lambda^{\star} \pi^{\star}}{n^{\star}}\right) \lambda^{\star} u^{\prime}\left(d^{\star}\right)-v\left(\lambda^{\star}\right) & =0
\end{aligned}
$$

In comparison with the previous section, there is here an additional FOC (35), concerning the optimal old-age labour $\lambda^{\star}$. It states that the old-age labour is optimal when the marginal gain in utility terms from working one more year (left-hand side) equals the marginal disutility from not retiring. Simplification yields

$$
u^{\prime}(d) F_{L}\left(k, 1+\frac{\lambda \pi}{n}\right)=v^{\prime}(\lambda)
$$

In comparison with the model with exogenous $\lambda$ [i.e. condition (22)], condition (37), which characterizes the optimum fertility rate, exhibits no explicit difference. However, it is likely to yield another optimum $n$, because consumptions and old-age labour take other values than in $(27)$, as $F_{L}\left(k, 1+\frac{\lambda \pi}{n}\right)=v^{\prime}(\lambda) / u^{\prime}(d)$ under the optimal $\lambda$.

Condition (38) characterizes the interior optimum survival rate. There is here an additional, negative term in comparison with condition (23). That term captures the negative effect of a higher survival rate on old-age utility given old-age labour. The condition for a corner solution $\pi^{\star}=1$ is now:

$$
u(d)-d u^{\prime}(d)+F_{L}\left(k, 1+\frac{\lambda \pi}{n}\right) \lambda u^{\prime}(d)-v(\lambda)>0
$$

The condition for the corner solution $\pi^{\star}=0$ becomes

$$
u(d)-d u^{\prime}(d)+F_{L}\left(k, 1+\frac{\lambda \pi}{n}\right) \lambda u^{\prime}(d)-v(\lambda)<0
$$

Finally, the second-order conditions for a maximum include now an additional condition, relative to the optimal level of old-age labour. Hence we have now five conditions. To save 
space, we only report here the first three ones.

$$
\begin{aligned}
\frac{\left(\pi^{\star}\right)^{2} u^{\prime \prime}\left(c^{\star}\right)}{n^{\star}}+n^{\star} \pi^{\star} u^{\prime \prime}\left(d^{\star}\right) & <0 \\
F_{K K}\left[\pi^{\star} u^{\prime \prime}\left(c^{\star}\right)+\left(n^{\star}\right)^{2} u^{\prime \prime}\left(d^{\star}\right)\right] & >0 \\
-\left[\left(\pi^{\star}\right)^{2} F_{K L}^{2} u^{\prime}\left(d^{\star}\right)\left(\pi^{\star} u^{\prime \prime}\left(c^{\star}\right)+\left(n^{\star}\right)^{2} u^{\prime \prime}\left(d^{\star}\right)\right)-F_{K K}\left(n^{\star} F_{L}^{2} u^{\prime \prime}\left(c^{\star}\right) u^{\prime \prime}\left(d^{\star}\right)\right)\right] & \\
+F_{K K}\left(\left(\pi^{\star}\right)^{2} F_{L L} u^{\prime}\left(d^{\star}\right)-n^{\star} \pi^{\star} v^{\prime \prime}\left(\lambda^{\star}\right)\right)\left(\pi^{\star} u^{\prime \prime}\left(c^{\star}\right)+\left(n^{\star}\right)^{2} u^{\prime \prime}\left(d^{\star}\right)\right) & <0
\end{aligned}
$$

Conditions (42) and (43) are the same as in the previous section, because the first-order conditions for optimal $d$ and $k$ are formally identical whatever $\lambda$ is exogenous or endogenous. However, condition (44) differs from its counterparts in the previous Section (i.e. expression (28)). Whereas conditions (42) and (43) are necessarily satisfied (thanks to the concavity of $u(\cdot)$ and $F(\cdot, \cdot)$ ), the same is not true for the other conditions. Only particular assumptions on the functional forms would allow us to say more about their fullfilment.

\subsection{The Serendipity Theorem}

Consider now the competitive economy. As before, factors are paid at their marginal productivities, i.e. (30) and (31) hold.

The young agent chooses his savings and old-age labour supply subject to his budget constraint. The problem of a young agent can be written as

$$
\max _{c, d, \lambda} u(c)+\pi u(d)-\pi v(\lambda)
$$

subject to the lifetime budget constraint (32).

From the FOCs, we have the Euler equation (16) and an additional condition related to labour supply:

$$
\pi v^{\prime}(\lambda)=u^{\prime}(c)\left(c+\pi \frac{d}{R}\right)\left(\frac{\pi}{R+\lambda \pi}\right)
$$

Finally, in equilibrium, capital equals savings, i.e. (17) holds.

For given $(n, \pi)$, a stationary competitive equilibrium is a vector $(\tilde{w}, \tilde{R}, \tilde{c}, \tilde{d}, \tilde{k}, \tilde{\lambda})$ satisfying (16), (17), (30)-(31), (32) and (45).

If $n$ is fixed at its optimum level, so that condition (37) holds, satisfied, then it follows that conditions (16), (17), (30)-(31), (32) imply the optimal rules (33)-(34) and (36).

However, in comparison with the previous section, we now have an additional condition characterizing the social optimum: condition (35), which characterizes the optimal old-age labour. Can this be obtained from the individual's decision, described by condition (45)? Actually, it can be shown that imposing the optimal values of demographic parameters $n$ 
and $\pi$ suffices to induce the optimal old-age working time. To see this, let us first substitute for $R=n$ and for (36) in expression (45):

$$
\pi v^{\prime}(\lambda)=u^{\prime}(c)\left(F\left(k, 1+\frac{\lambda \pi}{n}\right)-n k\right)\left(\frac{\pi}{n+\lambda \pi}\right)
$$

Given that $F\left(k, 1+\frac{\lambda \pi}{n}\right)=R k+w\left(1+\frac{\lambda \pi}{n}\right)$, and given (16), we have

$$
v^{\prime}(\lambda)=u^{\prime}(d) F_{L}\left(k, 1+\frac{\lambda \pi}{n}\right)
$$

which coincides with condition (35). Thus, imposing $\pi^{\star}$ and $n^{\star}$ suffices to decentralize the optimum retirement age. Endogenizing the old-age labour does not break the Extended Serendipity Theorem.

Proposition 3 Suppose that there exists a unique and stable steady-state equilibrium with a positive capital $k>0$.

Assume that the first order Conditions (33)-(37), (38) and the second order conditions for a maximum hold, then, if the government imposes $n^{\star}$ and $\pi^{\star}$, the economy will converge towards the optimum steady-state, which involves $0<n^{\star}<+\infty$ and $0<\pi^{\star}<1$.

Assume that the first order Conditions (33)-(37), (40) and the second order conditions for a maximum hold, then, if the government imposes $n^{\star}$ and $\pi^{\star}$, the economy will converge towards the optimum steady-state, which involves $0<n^{\star}<+\infty$ and $\pi^{\star}=1$.

Undoubtedly, Proposition 3 illustrates the robustness of the Extended Serendipity Theorem studied in the previous sections. Controlling for demographic conditions $\left(n^{\star}, \pi^{\star}\right)$ leads to the optimum steady-state not only in a world where agents either do not work when being old (Proposition 1) or do work during some fixed period (Proposition 2), but, also, in a world where agents organize their working lifeplans by themselves (Proposition 3). Hence the capacity to decentralize the optimum steady-state on the mere basis of demographic controls is robust to the endogenization of the retirement age. ${ }^{28}$

\section{Conclusion}

Despite the large attention that was paid to the economic environment (i.e. utility and production functions) under which Samuelson's Serendipity Theorem holds, little had been said on the conditions of the demographic environment guaranteeing that result. The present paper aimed at re-examining whether the Serendipity Theorem still holds under a richer demographic structure.

\footnotetext{
${ }^{28}$ However, that robustness of the Extended Serendipity Theorem remains conditional on the particular way in which old-age labour is modelled in our framework. The present work has thus no pretension to exhaustiveness, and could be complemented by additional robustness studies (see below).
} 
For that purpose, we developed a two-period OLG model with risky lifetime, and showed that, if there exists only one stable steady-state, then, provided the government can impose the optimum levels of the fertility rate and of the survival rate, all other economic variables will take their optimum values, that is, the optimum optimorum will be reached. That result was also shown to hold - but with different targets - in a more general framework, where adults surviving to the old age are allowed to work a fraction of the second period, whatever that fraction is exogenously fixed or endogenously chosen by agents.

Hence, the introduction of mortality in a Diamond-type economy does not infirm the Serendipity Theorem, as the imposition of the optimum fertility rate is still beneficial here, even though this is no longer sufficient to reach the optimum optimorum, which requires also the optimum survival rate to be imposed. This paper tends thus to emphasize, in some sense, a limitation of the Serendipity Theorem, as one cannot, by the use of a single demographic control, reach the best steady-state: longevity must also be controlled for, and its level affects the optimum level of fertility. The two demographic dimensions must thus be treated together.

Finally, it should be stressed that the extended version of the Serendipity Theorem considered here relies on several assumptions, which should be relaxed in future works. There are three major assumptions to be discussed further. Firstly, the possibility to keep the Serendipity Theorem in the context of risky lifetime relies on the existence of a perfect annuity market. Without it, the question of whether fixing the optimal pair $\left(n^{\star}, \pi^{\star}\right)$ suffices to induce the optimum steady-state would become more complex. Given the under-development of annuity markets in real life, a natural direction for future research would consist of reconsidering the Serendipity Theorem under alternative assumptions on the savings of the dead. Secondly, one may also want to explore the consequences from relaxing the average utilitarian social welfare function postulated throughout this paper. Actually, the population ethics literature emphasized the difficulties, when facing different-numbers choices, to adhere to a particular social objective. Hence exploring the robustness of the Serendipity Theorem to various definitions of the social objective is a natural direction for future works. ${ }^{29}$ Thirdly, the introduction of costs for controlling fertility and survival would also be most welcome, since in reality the control of demographic variables is definitely costly. Note that relaxing those three assumptions would involve significant departures from Samuelson's approach, and, also, much additional complexity. Therefore those robustness checks are left for future research.

\section{References}

Abio, G. (2003): 'Interiority of the optimal population growth rate with endogenous fertility', Economics Bulletin, 10(4), pp. 1-7.

\footnotetext{
${ }^{29}$ Note that, more generally, additional discussions on the normative foundations of Samuelson's approach would also be welcome, since one may accuse such an instrumental use of demographic variables of excess normativism. But such discussions on the normative foundations of population economics would require an entire paper on their own, and are thus left for future research.
} 
Abio, G., Mahieu, C., \& Paxtot, C. (2004): 'On the optimality of PAYG pension systems in an endogenous fertility setting', Journal of Pensions Economics and Finance, 3(1), pp. 35-62.

Becker, G. \& Philipson, T. (1998): 'Old-age longevity and mortality contingent claims', The Journal of Political Economy, 106(3), pp. 551-573.

Blackburn, K. \& Cipriani, G.P. (2002): 'A model of longevity, fertility and growth', Journal of Economic Dynamics and Control, 26, pp. 187-204.

Blackorby, C., Bossert, W. \& Donaldson, D. (2005): Population Issues in Social Choice Theory, Welfare Economics and Ethics. Econometric Society Monographs, Cambridge University Press.

Brown, J.R. (2007): 'Rational and behavioural perspectives on the role of annuities in retirement planning', NBER Working Paper 13537.

Cervellati M. \& Sunde, U. (2007): 'Fertility, Education, and Mortality: A Unified Theory of the Economic and Demographic Transition.' mimeo, IZA, Bonn.

Chakraborty, S. (2004): 'Endogenous lifetime and economic growth', Journal of Economic Theory, 116, pp. 119-137.

Deardorff, A.V. (1976): 'The optimum growth rate for population: comment', International Economic Review, 17(2), pp. 510-515.

de la Croix, D., Mahieu, G. \& Rillaers, A. (2004): 'How should the allocation of resources adjust to the baby bust?', Journal of Public Economic Theory, 6(4), pp. 607-636.

de la Croix, D. \& Michel, P. (2002): A Theory of Economic Growth. Dynamics and Policy in Overlapping Generations. Cambridge University Press.

de la Croix, D. \& Licandro, O. (2007): 'The Child is the Father of Man', CORE Discussion Paper 2007-72.

de la Croix, D. \& Ponthiere, G. (2010): 'On the Golden Rule of capital accumulation under endogenous longevity', Mathematical Social Sciences, 59(2), pp. 227-238.

Diamond, P. (1965): 'National debt in an neoclassical growth model', American Economic Review, 55(5), pp. 1126-1150.

Doepke, M. (2004): 'Accounting for Fertility Decline During the Transition to Growth' Journal of Economic Growth, 9, pp. 347-383.

Galor, O., \& Moav, O. (2005): 'Natural selection and the evolution of life expectancy', CEPR, Minerva Center for Economic Growth Paper 02-05.

$\mathrm{Hu}$, S. (1979): 'Social security, the supply of labor, and capital accumulation', American Economic Review, 69, pp. 274-283.

Jaeger, K. \& Kuhle, W. (2009): 'The optimum growth rate for population reconsidered', Journal of Population Economics, 22, pp. 23-41.

Jouvet, P.A., Pestieau, P. \& Ponthiere, G. (2010): 'Longevity and environmental quality in an OLG model', Journal of Economics, 100(3), pp. 191-216. 
Michel, P. \& Pestieau, P. (1993): 'Population growth and optimality: when does the serendipity hold', Journal of Population Economics, 6(4), pp. 353-362.

Parfit, D. (1984): Reasons and Persons. Oxford University Press.

Ponthiere, G. (2009): 'Rectangularization and the rise of limit-longevity in a simple overlapping generations model', The Manchester School, 77(1), pp. 17-46.

Samuelson, P. (1975): 'The optimum growth rate for population', International Economic Review, 16(3), pp. 531-538.

\section{Appendix}

\subsection{The CIES example}

Let us now see in which particular cases of temporal utility function condition (6) could be satisfied. For instance, under Constant Intertemporal Elasticity of substitution (CIES) utility function,

$$
u(d)=\frac{(d)^{1-\sigma}}{1-\sigma}+\alpha
$$

we have:

$$
u(d)-d u^{\prime}(d)=\frac{(d)^{1-\sigma}}{1-\sigma}+\alpha-(d)^{1-\sigma}
$$

The RHS is zero only if

$$
\begin{aligned}
\frac{(d)^{1-\sigma}}{1-\sigma}+\alpha & =(d)^{1-\sigma} \\
\alpha & =(d)^{1-\sigma}\left(\frac{-\sigma}{1-\sigma}\right)
\end{aligned}
$$

That condition states that, for a positive or zero level of $\alpha$, the intercept of the temporal utility function, the optimal survival rate is not an interior solution, but is a corner solution, with $\pi^{\star}=1$, as we have condition (7), i.e. $u(d)-d u^{\prime}(d)>0$, so that condition (6) cannot be satisfied. Thus, it is only under a particular, negative $\alpha$ that condition (6) can be satisfied. Clearly, $\alpha$ cannot be positive, but it cannot be too negative either: if $\alpha$ is smaller than the threshold, we have condition (8), i.e. $u(d)-d u^{\prime}(d)<0$, so that the optimal $\pi$ is a corner solution, and equals 0 .

\subsection{Second-order conditions}

The Hessian matrix of the second-order derivatives associated to the planner problem of Section 3 is 


$$
H \equiv\left[\begin{array}{cccc}
\frac{\partial^{2} U}{\partial d^{2}} & \frac{\partial U^{2}}{\partial d \partial k} & \frac{\partial U^{2}}{\partial d \partial n} & \frac{\partial U^{2}}{\partial d \partial \pi} \\
\frac{\partial U^{2}}{\partial k \partial d} & \frac{\partial^{2} U}{\partial k^{2}} & \frac{\partial U^{2}}{\partial k \partial n} & \frac{\partial U^{2}}{\partial k \partial \pi} \\
\frac{\partial U^{2}}{\partial n \partial d} & \frac{\partial U^{2}}{\partial n \partial k} & \frac{\partial^{2} U}{\partial n^{2}} & \frac{\partial U^{2}}{\partial n \partial \pi} \\
\frac{\partial U^{2}}{\partial \pi \partial d} & \frac{\partial U^{2}}{\partial \pi \partial k} & \frac{\partial U^{2}}{\partial \pi \partial n} & \frac{\partial^{2} U}{\partial \pi^{2}}
\end{array}\right]
$$

Hence,

$$
u^{\prime \prime}(c)\left[\begin{array}{cccc}
\frac{\pi^{2}}{n^{2}}+\pi \frac{u^{\prime}(d)}{u^{\prime \prime}(c)} & \frac{\pi\left(n-f^{\prime}\right)}{n} & \frac{\pi u^{\prime}(c)}{u^{\prime \prime}(c) n^{2}}-\frac{\pi\left(\frac{\pi d}{n^{2}}-k\right)}{n} & \frac{u^{\prime}(d)}{u^{\prime \prime}(c)}-\frac{u^{\prime}(c)}{u^{\prime \prime}(c) n}+\frac{d \pi}{n^{2}} \\
\frac{\pi\left(n-f^{\prime}\right)}{n} & \left(f^{\prime}-n\right)^{2}+\frac{u^{\prime}(c)}{u^{\prime \prime}(c)} f^{\prime \prime} & \left(\frac{\pi d}{n^{2}}-k\right)\left(f^{\prime}-n\right)-\frac{u^{\prime}(c)}{u^{\prime \prime}(c)} & \frac{d\left(n-f^{\prime}\right)}{n} \\
\frac{\pi u^{\prime}(c)}{n^{2} u^{\prime \prime}(c)}-\frac{\pi\left(\frac{\pi d}{n} n^{2}\right.}{n} & \left(\frac{\pi d}{n^{2}}-k\right)\left(f^{\prime}-n\right)-\frac{u^{\prime}(c)}{u^{\prime \prime}(c)} & -\frac{2 d \pi u^{\prime}(c)}{n^{3} u^{\prime \prime}(c)}+\left(\frac{\pi d}{n^{2}}-k\right)^{2} & \frac{d u^{\prime}(c)}{n^{2} u^{\prime \prime}(c)}-\frac{d\left(\frac{\pi d}{n^{2}}-k\right)}{n} \\
\frac{u^{\prime}(d)}{u^{\prime \prime}(c)}-\frac{u^{\prime}(c)}{u^{\prime \prime}(c) n}+\frac{d \pi}{n^{2}} & \frac{d\left(n-f^{\prime}\right)}{n} & \frac{d u^{\prime}(c)}{n^{2} u^{\prime \prime}(c)}-\frac{d\left(\frac{\pi d}{n^{2}}-k\right)}{n} & \frac{d^{2}}{n^{2}}
\end{array}\right]
$$

Substituting for first-order conditions and multiplying by $n$ yields:

$$
\left[\begin{array}{cccc}
\frac{u^{\prime \prime}(c) \pi^{2}}{n}+n \pi u^{\prime \prime}(d) & 0 & \pi u^{\prime}(d) & \frac{\pi u^{\prime \prime}(c) d}{n} \\
0 & n^{2} u^{\prime}(d) f^{\prime \prime}(k) & -n^{2} u^{\prime}(d) & 0 \\
\pi u^{\prime}(d) & -n^{2} u^{\prime}(d) & -\frac{2 d \pi u^{\prime}(d)}{n} & d u^{\prime}(d) \\
\frac{\pi u^{\prime \prime}(c) d}{n} & 0 & d u^{\prime}(d) & \frac{d^{2} u^{\prime \prime}(c)}{n}
\end{array}\right]
$$

A sufficient condition for $(d, k, n, \pi)$ to be a maximum is that the Hessian matrix is negative definite. Four conditions guarantee that the Hessian matrix is negative definite. Those conditions concern the determinants of the four submatrices, of sizes $1 \times 1,2 \times 2,3 \times 3$ and $4 \times 4$. Those conditions are conditions (9)-(12) in Section $3 .{ }^{30}$

\subsection{The planner's problem: an analytical example}

Let us now illustrate the general model of Section 3 by imposing some functional forms for $u(\cdot)$ and $f(\cdot)$.

For that purpose, we assume a standard CIES utility function with intercept:

$$
u(c)=\frac{(c)^{1-\sigma}}{1-\sigma}+\alpha
$$

Moreover, we assume a Cobb-Douglas production function:

$$
f(k)=A k^{\gamma}
$$

where $\gamma$ is the elasticity of output per worker with respect to capital per worker $(0<\gamma<1)$.

\footnotetext{
${ }^{30}$ Note that it would be ideal to provide a representation of those SOCs in line with the treatment proposed by Jaeger and Kuhle (2009). However, our problem includes one additional dimension, which prevents us from providing such a treatment of SOCs.
} 
Substituting for $u(\cdot)$ and $f(\cdot)$ in FOCs yields:

$$
\begin{aligned}
\frac{c^{-\sigma}}{d^{-\sigma}} & =n \\
A \gamma k^{\gamma-1} & =n \\
A k^{\gamma}-n k & =c+\pi \frac{d}{n} \\
\pi \frac{d}{n^{2}} & =k \\
(d)^{1-\sigma}\left(1-\frac{1}{1-\sigma}\right) & =\alpha
\end{aligned}
$$

Substituting for $u(\cdot)$ and $f(\cdot)$ in SOCs yields:

$$
\begin{gathered}
-\frac{\pi^{2} \sigma c^{-\sigma-1}}{n}-n \pi \sigma d^{-\sigma-1}<0 \\
n \pi d^{-\sigma} A \gamma(\gamma-1) k^{\gamma-2}\left[-\pi \sigma c^{-\sigma-1}-n^{2} \sigma d^{-\sigma-1}\right]>0 \\
\frac{\pi}{d^{\sigma}}\left[\frac{n^{2} \pi A \gamma(\gamma-1) k^{\gamma-2}}{d^{2 \sigma}}+\frac{n^{2}\left[\pi\left(n+2 k A \gamma(\gamma-1) k^{\gamma-2}\right)\left(-\sigma c^{-\sigma-1}\right)\right]}{d^{\sigma}}\right]+\frac{\pi\left(n^{3}+2 d \pi A \gamma(\gamma-1) k^{\gamma-2}\right) \sigma}{d^{2 \sigma+1}}<0 \\
-\frac{n^{3} \pi}{d^{2 \sigma-2}}\left[\frac{A \gamma(\gamma-1) k^{\gamma-2}}{d^{\sigma}}-\frac{\left(n+2 k A \gamma(\gamma-1) k^{\gamma-2}\right)}{\left(\sigma c^{\sigma+1}\right)}\right]\left(-\sigma d^{-\sigma-1}\right)>0
\end{gathered}
$$

It is straightforward to see that the first two SOCs are satisfied, given $\gamma<1$ and $\sigma>0$. Regarding the third SOC, this can be rewritten as:

$$
\begin{gathered}
n^{2} \pi^{2} d^{-3 \sigma} A \gamma(\gamma-1) k^{\gamma-2}-n^{3} d^{-2 \sigma} \pi^{2} \sigma c^{-\sigma-1}-n^{2} d^{-2 \sigma} 2 \pi^{2} A \gamma(\gamma-1) k^{\gamma-1} \sigma c^{-\sigma-1}+n^{3} \pi \sigma d^{-2 \sigma-1} \\
+2 \pi^{2} A \gamma(\gamma-1) k^{\gamma-2} \sigma d^{-2 \sigma}<0
\end{gathered}
$$

The first and second terms are negative, the third term is positive, like the fourth term, whereas the fifth term is negative. That condition is satisfied if and only if

$$
\begin{gathered}
n^{2} d^{-2 \sigma} 2 \pi^{2} A \gamma(1-\gamma) k^{\gamma-1} \sigma c^{-\sigma-1}+n^{3} \pi \sigma d^{-2 \sigma-1} \\
<n^{2} \pi^{2} d^{-3 \sigma} A \gamma(1-\gamma) k^{\gamma-2}+n^{3} d^{-2 \sigma} \pi^{2} \sigma c^{-\sigma-1}+2 \pi^{2} A \gamma(1-\gamma) k^{\gamma-2} \sigma d^{-2 \sigma}
\end{gathered}
$$

Substituting for the FOC $A \gamma k^{\gamma-1}=n$, we get:

$$
\begin{aligned}
& n^{2} 2 \pi(1-\gamma) \sigma c^{-\sigma-1}+n^{2} \sigma d^{-1} \\
< & n^{2} \pi d^{-\sigma}(1-\gamma) k^{-1}+n^{2} \pi \sigma c^{-\sigma-1}+2 \pi(1-\gamma) k^{-1} \sigma
\end{aligned}
$$


Using $\pi \frac{d}{n^{2}}=k$, and assuming $\pi>0$, we obtain:

$$
\pi \sigma c^{-\sigma-1}(1-2 \gamma)<n^{2} d^{-\sigma-1}(1-\gamma)+\sigma d^{-1}(1-2 \gamma)
$$

Note that this inequality is always satisfied when $\sigma$ tends towards 0 . Note also, that, when $\gamma$ tends towards $1 / 2$, we have: $0<n^{2} d^{-\sigma-1}(1-\gamma)$, which is always true.

Finally, the fourth SOC can be written as:

$$
n^{3} \pi \sigma d^{-4 \sigma+1} A \gamma(\gamma-1) k^{\gamma-2}-n^{4} \pi d^{-3 \sigma+1} \sigma^{2} c^{-\sigma-1}+n^{3} \pi \sigma^{2} d^{-3 \sigma+1} c^{-\sigma-1} 2 k A \gamma(1-\gamma) k^{\gamma-2}>0
$$

The first and second terms are negative, but the third term is positive. Hence that SOC is satisfied if and only if

$$
n^{3} \pi \sigma^{2} d^{-3 \sigma+1} c^{-\sigma-1} 2 k A \gamma(1-\gamma) k^{\gamma-2}>n^{3} \pi \sigma d^{-4 \sigma+1} A \gamma(1-\gamma) k^{\gamma-2}+n^{4} \pi d^{-3 \sigma+1} \sigma^{2} c^{-\sigma-1}
$$

Using FOCs $A \gamma k^{\gamma-1}=n$ and $\pi \frac{d}{n^{2}}=k$, that inequality can be written as:

$$
\pi>\frac{d^{-\sigma-1}(1-\gamma) n^{2}}{\sigma c^{-\sigma-1}(1-2 \gamma)}
$$

That inequality requires $\sigma$ to be sufficiently large. Note that this inequality is always true when $\gamma>1 / 2$, but may fail to be true under $\gamma \leq 1 / 2$.

To sum up, whereas the first and second SOCs are always satisfied thanks to the concavity of $u(\cdot)$ and $f(\cdot)$, things are more complicated for the third and fourth FOC. The third SOC is more likely to be satisfied for a low $\sigma$, whereas the opposite is true for the fourth FOC. Hence it is only for an intermediate level of $\sigma$ that both SOCs can be satisfied. Finally, it should also be stressed that the elasticity of output with respect to capital $\gamma$ plays an important role regarding the validity of the SOCs.

\subsection{Second-order conditions in Section 4}

Substituting for first-order conditions in the Hessian matrix yields:

$$
u^{\prime}(d)\left[\begin{array}{cccc}
\frac{u^{\prime \prime}(c) \pi^{2}}{u^{\prime}(d) n}+n \pi \frac{u^{\prime \prime}(d)}{u^{\prime}(d)} & 0 & -\frac{\pi F_{L} u^{\prime \prime}(c)}{u^{\prime}(d) n} & \pi \\
0 & n^{2} F_{K K} & n \pi F_{K L} & -n^{2}-\pi \lambda F_{K L} \\
-\frac{\pi u^{\prime \prime}(c) F_{L}}{u^{\prime}(d) n} & n \pi F_{L K} & \frac{F_{L}^{2} u^{\prime \prime}(c)}{n}+\pi^{2} F_{L L} & -\frac{n F_{L}}{n}-\frac{\pi^{2} \lambda F_{L L}}{n} \\
\pi & -n^{2}-\pi \lambda F_{K L} & -\frac{n F_{L}}{n}-\frac{\pi^{2} \lambda F_{L L}}{n} & \frac{-2 k n^{3}}{n^{2}}+\frac{\pi^{2} \lambda^{2} F_{L L}}{n^{2}}
\end{array}\right]
$$

Given that the FOCs for optimal $d$ and $k$ are exactly identical to the ones under no oldage labour, this is not surprising that the $2 \times 2$ submatrix is the same as before. However, the FOCs for optimal $n$ and $\pi$ are different from the ones in Section 3, and this has some consequences on the $3 \times 3$ and $4 \times 4$ submatrices. As above, four conditions guarantee that the Hessian matrix is negative definite. Those conditions concern the determinants of the four submatrices, of sizes $1 \times 1,2 \times 2,3 \times 3$ and $4 \times 4$. Those conditions are conditions (26)-(29) in Section 4. 


\subsection{Second-order conditions in Section 5}

The Hessian matrix is now a $5 \times 5$ matrix

$$
\left[\begin{array}{ccccc}
\frac{\partial^{2} U}{\partial d^{2}} & \frac{\partial U^{2}}{\partial d \partial k} & \frac{\partial U^{2}}{\partial d \partial n} & \frac{\partial U^{2}}{\partial d \partial \pi} & \frac{\partial U^{2}}{\partial d \partial \lambda} \\
\frac{\partial U^{2}}{\partial k \partial d} & \frac{\partial^{2} U}{\partial k^{2}} & \frac{\partial U^{2}}{\partial k \partial n} & \frac{\partial U^{2}}{\partial k \partial \pi} & \frac{\partial U^{2}}{\partial k \partial \lambda} \\
\frac{\partial U^{2}}{\partial n \partial d} & \frac{\partial U^{2}}{\partial n \partial k} & \frac{\partial^{2} U}{\partial n^{2}} & \frac{\partial U^{2}}{\partial n \partial \pi} & \frac{\partial U^{2}}{\partial n \partial \lambda} \\
\frac{\partial U^{2}}{\partial \pi \partial d} & \frac{\partial U^{2}}{\partial \pi \partial k} & \frac{\partial U^{2}}{\partial \pi \partial n} & \frac{\partial^{2} U}{\partial \pi^{2}} & \frac{\partial U^{2}}{\partial \pi \partial \lambda} \\
\frac{\partial U^{2}}{\partial \lambda \partial d} & \frac{\partial U^{2}}{\partial \lambda \partial k} & \frac{\partial U^{2}}{\partial \lambda \partial n} & \frac{\partial U^{2}}{\partial \lambda \partial \pi} & \frac{\partial U^{2}}{\partial \lambda^{2}}
\end{array}\right]
$$

Substituting for the first-order conditions in the entries and simplifying yields

$$
\left[\begin{array}{ccc}
\frac{u^{\prime \prime}(c) \pi^{2}}{n}+n \pi u^{\prime \prime}(d) & 0 & -\frac{\pi F_{L} u^{\prime \prime}(c)}{n} \\
0 & n^{2} u^{\prime}(d) F_{K K} & n \pi F_{K L} u^{\prime}(d) \\
-\frac{\pi u^{\prime \prime}(c) F_{L}}{n} & n \pi F_{L K} u^{\prime}(d) & \frac{F_{L}^{2} u^{\prime \prime}(c)}{n}-\pi n v^{\prime \prime}(\lambda)+\pi\left(\pi F_{L L} u^{\prime}(d)\right) \\
\pi u^{\prime}(d) & -u^{\prime}(d) n^{2}-u^{\prime}(d) \pi \lambda F_{K L} & -u^{\prime}(d) \frac{n F_{L}}{n}-u^{\prime}(d) \frac{\pi^{2} \lambda F_{L L}}{n} \\
u^{\prime \prime}(c) \frac{d \pi}{n}-u^{\prime \prime}(c) \frac{\lambda F_{L}}{n} & n \lambda F_{K L} u^{\prime}(d) & \frac{\lambda F_{L}^{2} u^{\prime \prime}(c)+n \pi^{2} \lambda F_{L L} u^{\prime}(d)}{n \pi}-\frac{F_{L}\left[d \pi u^{\prime \prime}(c)+n^{2}(\pi-1) u^{\prime}(d)\right]}{n \pi} \\
-u^{\prime}(d) n^{2}-u^{\prime}(d) \pi \lambda F_{K L} & u^{\prime \prime}(c) \frac{d \pi}{n}-u^{\prime \prime}(c) \frac{\lambda F_{L}}{n} \\
-u^{\prime}(d) \frac{n F_{L}}{n}-u^{\prime}(d) \frac{\pi^{2} \lambda F_{L L}}{n} & \frac{\lambda F_{L}^{2} u^{\prime \prime}(c)+n \pi^{2} \lambda F_{L L} u^{\prime}(d)}{n \pi}-\frac{F_{L}\left[d \pi u^{\prime \prime}(c)+n^{2}(\pi-1) u^{\prime}(d)\right]}{n \pi} \\
u^{\prime}(d) \frac{-2 k n^{3}}{n n^{2}}+u^{\prime}(d) \frac{\pi^{2} \lambda^{2} F_{L L}}{n{ }^{2}} & u^{\prime}(d) \frac{d n-\frac{n \lambda F_{L}}{n}}{n}-u^{\prime}(d) \frac{\pi \lambda^{2} F_{L L}}{n} \\
u^{\prime}(d) \frac{d n-\frac{n \lambda F_{L}}{\pi}}{n}-u^{\prime}(d) \frac{\pi \lambda^{2} F_{L L}}{n} & \frac{\left(d-\frac{\lambda F_{L}}{\pi}\right)^{2} u^{\prime \prime}(c)}{n}+\frac{n \lambda^{2} F_{L L} u^{\prime}(d)}{n}
\end{array}\right]
$$

Five conditions guarantee that the Hessian matrix is negative definite. Those conditions concern the determinants of the five submatrices, of sizes $1 \times 1,2 \times 2,3 \times 3,4 \times 4$ and $5 \times 5$. The first three of them are conditions (42)-(44) in Section 5. 